\title{
Caracterización bromatología, microbiológica y sensorial de la salchicha Frankfurt elaborada con Citrato de Calcio como antioxidante natural
}

Bromatological, microbiological and sensory characterization of the Frankfurt sausage made with Calcium Citrate as a natural antioxidant

Fabricio Armando Guzmán Acán. 1, Verónica de Los Ángeles Bonifaz Ramos. ${ }^{2}$, Cristian José Esparza Bonilla. ${ }^{3}$

\begin{abstract}
.
DOI: https://doi.org/10.33262/cienciadigital.v3i3.663

In the meat production center of the $\mathrm{ESPOCH}$, different levels of calcium citrate from the eggshells were evaluated as an antioxidant (0.1, 0.2 and $0.3 \%)$ in the Frankfurt type sausage elaboration, with three replications per treatment and an experimental unit size of $8 \mathrm{Kg}$ containing beef, pork, pig fat, ice, additives and condiments. The experimental results were distributed under a completely at random design. It was determined that the nutritive quality was not statistically affected by the calcium citrate addition. Mesophyll aerobe and total colliform loads were not found in all treatments differences between the reported values, considering that all treatments were acceptable for the consumer obtaining a high mark of very good. In rack life all formulations surpassed 15 days refrigeration. With the three calcium citrate treatments the production costs are reduced as to the control (3.72-3.68), 1.29 USD cost-benefit with the control and 1.31
\end{abstract}

1 Escuela Superior Politécnica de Chimborazo, Facultad de Ciencias, Chimborazo, Ecuador. fabricio.guzman@espoch.edu.ec

2 Consultor Serinamb CIA, Chimborazo, Ecuador veronicabonifaz@gmail.com

3 Escuela Superior Politécnica de Chimborazo, Unidad de Admisión y Nivelación cesparza@espoch.edu.ec 
with the rest of treatments. It is therefore recommended to use any of these formulations.

Keywords: Calcium Citrate, egg shell, Frankfurt sausage, antioxidant, sausages

\section{Resumen.}

En el Centro de Producción de Cárnicos de la ESPOCH, se evaluó diferentes niveles de citrato de calcio obtenido de las cáscaras de huevos como antioxidante $(0.1,0.2$ y $0.3 \%$ ) en la elaboración de salchicha tipo Frankfurt, con tres repeticiones por tratamiento y un tamaño de la unidad experimental de $8 \mathrm{~kg}$ que contenían carne de res, carne de cerdo, grasa de cerdo, hielo, aditivos y condimentos. Determinándose que la calidad nutritiva no se vio afectada estadísticamente por efecto de la adición de citrato de calcio. No se encontró cargas de aerobios mesófilos y coliformes totales, en todos los tratamientos, considerándose un alimento apto para el consumo humano. Las características organolépticas presentaron diferencias entre los valores reportados, considerándose todos los tratamientos aceptables para el consumidor obteniendo una calificación de muy buena. En la vida de anaquel, todas las formulaciones sobrepasaron los 15 días de refrigeración. Con los tres tratamientos de citrato de calcio los costos de producción se reducen con respecto al testigo (3.72 a 3.68), un beneficio/costo de 1.29 dólares con el testigo y de 1.31 con el resto de tratamientos, por lo que se recomienda emplear cualquiera de estas formulaciones.

Palabras claves: Citrato de Calcio, cáscara de huevo, salchicha Frankfurt, antioxidante, embutidos

\section{Introducción.}

El hombre desde tiempos inmemorables ha ido buscando formas y métodos para conservar sus alimentos por largos periodos de tiempo, de esta incansable búsqueda el hombre ha logrado descubrir varios procesos para lograr este objetivo. La elaboración de productos derivados de la carne como los embutidos es uno de estos procedimientos, el cual se desarrolló durante un largo tiempo, empezando desde un simple proceso de secado y salazón de la carne fresca para ser consumida luego de un periodo de tiempo.

Con el desarrollo de la industria alimenticia cárnica, se dio principio al aparecimiento de métodos de conservación y elaboración de productos cárnicos con gran dependencia y utilización de conservantes, aditivos, y/o especias de origen químico, los cuales van cambiando las características naturales del producto elaborado, esto ha sido causa de que los alimentos no cumplan con su función principal la cual es la de brindar los nutrientes necesarios para el desarrollo normal de la vida en las personas que lo consumen, provocando que a la larga vayan produciendo complicaciones en la salud de los consumidores.

En vista de este inconveniente, en la actualidad se ha visto la necesidad de ir sustituyendo estos compuestos químicos, por otros de origen natural que aseguren obtener productos de excelente calidad, como es el caso del citrato de calcio un compuesto obtenido de la 
combinación de las cáscaras de huevos y el jugo de limón, que cumple la función de antioxidante de las grasas en los embutidos, al ser un compuesto natural, asegura a los consumidores de los productos cárnicos un alimento completo tanto en requerimientos nutricionales, como en el cuidado de su salud y el de su familia.

Además permite mantener los costos de elaboración de los productos cárnicos en niveles aceptables que le permitan al empresario de la industria cárnica competir en el mercado, sin el riesgo que representa en la mayoría de los casos la sustitución de los compuestos químicos por naturales como lo es una subida en los costos de producción, lo cual representa menores márgenes de ganancia.

Actualmente la prioridad que tienen las personas, es la de lograr sustituir por completo la utilización de productos de origen químico por aquellos de origen natural, en la elaboración de toda clase de alimentos en este caso los embutidos.

En vista de esta necesidad surge la presente investigación la cual consiste en sustituir antioxidantes de origen químico por compuesto naturales los cuales cumplan el mismo papel de antioxidante de las grasas en el embutido, en este caso por el compuesto denominado Citrato de Calcio, el cual se obtiene de la combinación de dos elementos naturales como son las cáscaras de huevos y el jugo de limón.

Logrando así encontrar un nuevo compuesto que a más de conservar de excelente manera el producto, asegure al consumidor la ingesta de alimentos que no comprometan su salud a corto ni a largo plazo.

Por lo anotado, en el presente trabajo se plantearon los siguientes objetivos:

- Obtener el citrato de calcio de las cáscaras de huevos y utilizarlo como antioxidante natural en distintas dosis $(0.1,0.2,0.3 \%)$ en la elaboración de salchicha Frankfurt

- Determinar el nivel adecuado de citrato de calcio en la elaboración de la salchicha Frankfurt, mediante la evaluación de las características bromatológicas, microbiológicas y organolépticas.

- Determinar los costos de producción y su rentabilidad mediante el indicador beneficio/costo $(\mathrm{B} / \mathrm{C})$

\section{Metodologia.}

En la planta de cárnicos de la FCP-ESPOCH, ubicada en la ciudad de Riobamba en la Panamericana Sur Km 1 1 $\frac{1}{2}$, Se evaluó la utilización de diferentes niveles de Citrato de Calcio $(0.1,0.2,0.3 \%)$, obtenidos de cáscaras de huevos, en la elaboración de salchicha Frankfurt para ser comparada con las salchichas obtenidas con la utilización de Eritorbato de sodio, se emplearon cuatro tratamientos experimentales con tres repeticiones en dos ensayos consecutivos. Los resultados experimentales obtenidos sometidos a análisis de varianza para las diferencias (ADEVA), separación de medias según la prueba de Waller - Duncan al nivel de significancia $\mathrm{P} \leq 0.05$ y $\mathrm{P} \leq 0.01$, en los parámetros bromatológicos. Estadística descriptiva 
para las variables del análisis microbiológico. Prueba de Rating Test (Witting, 1981), en variables no paramétricas del análisis organoléptico.

\section{Procedimiento Experimental}

- Para la obtención del citrato de calcio, se siguió el siguiente procedimiento.

- Lavar bien las cáscaras de huevo, quitarles la membrana de queratina y dejarlas secar.

- Pesar las cáscaras completamente secas utilizando una balanza digital.

- Moler las cáscaras utilizando un molino de mano.

- En el frasco esterilizado mezclar las cáscaras de huevo ya molidas con el jugo de limón y dejar reposar por 2 horas.

- Para quitar la humedad se calienta a fuego lento, hasta secar por completo el compuesto formado.

- Realizado esto se obtuvo el citrato de calcio el cual se utilizó en el estudio.

En el cuadro 1 se reportan las formulaciones para la elaboración de la salchicha Frankfurt.

Cuadro1.Formulacion de la salchicha frankfurt con diferentes niveles de citrato de calcio obtenido de las cáscaras de huevo y utilizado como antioxidante natural.

\begin{tabular}{llllll}
\hline & Referencia & \multicolumn{4}{l}{ Niveles de Citrato de Calcio, $\%$} \\
\hline Insumos & $(\%)$ & 0 & 0,1 & 0,2 & 0,3 \\
Carne de res & 60,000 & 4,800 & 4,800 & 4,800 & 4,800 \\
Carne de cerdo & 20,000 & 1,600 & 1,600 & 1,600 & 1,600 \\
Grasa de cerdo & 20,000 & 1,600 & 1,600 & 1,600 & 1,600 \\
Aditivos & & & & & \\
Sal & 2,200 & 0,176 & 0,176 & 0,176 & 0,176 \\
Sal nitro & 0,025 & 0,002 & 0,002 & 0,002 & 0,002 \\
Fosfato & 0,300 & 0,024 & 0,024 & 0,024 & 0,024 \\
Eritorbato de sodio & 0,080 & 0,006 & 0,000 & 0,000 & 0,000 \\
Condimento & 0,500 & 0,040 & 0,040 & 0,040 & 0,040 \\
Hielo & 25,000 & 2,000 & 2,000 & 2,000 & 2,000 \\
Azúcar & 0,200 & 0,016 & 0,016 & 0,016 & 0,016 \\
Leche & 0,200 & 0,016 & 0,016 & 0,016 & 0,016 \\
Pimienta blanca & 0,300 & 0,024 & 0,024 & 0,024 & 0,024 \\
Ajo polvo & 0,200 & 0,016 & 0,016 & 0,016 & 0,016 \\
Sorbato & 0,010 & 0,001 & 0,001 & 0,001 & 0,001 \\
Citrato de calcio & 0,000 & 0,000 & 0,008 & 0,016 & 0,024 \\
Total, kg & & 10,321 & 10,323 & 10,331 & 10,339 \\
\hline Fuente: Mira, J. & & & & &
\end{tabular}

Fuente: Mira, J. (1998). 
El proceso de elaboración fue el siguiente: Una vez troceada la materia prima se procedió a moler tanto la grasa como las carnes, luego se puso en el cutter para realizar la emulsión, donde que se le fue añadiendo el hielo para estabilizar la emulsión, así como los respectivos aditivos y el compuesto en estudio; luego se realizó la operación de embutido en tripas sintéticas de $16 \mathrm{~mm}$ de diámetro y se fue dividiendo a un largo aproximado de $12 \mathrm{~cm}$, para luego proceder a cocerla. Posteriormente se las duchó con agua fría y se las puso en refrigeración hasta su comercialización.

Cuadro 2. Esquema del Experimento por Replica.

\begin{tabular}{|c|c|c|c|c|c|c|}
\hline \multirow[t]{2}{*}{ Tratamientos } & \multirow[t]{2}{*}{ Código } & \multirow[t]{2}{*}{ Rept. } & \multicolumn{2}{|l|}{ TUE* } & \multicolumn{2}{|c|}{ Total/Tratamiento } \\
\hline & & & Elaborac. & Análisis & Elaborac. & Análisis \\
\hline Eritorbato de sodio & T0 & 3 & $8 \mathrm{~kg}$ & $120 \mathrm{~g}$ & $24 \mathrm{~kg}$ & $300 \mathrm{~g}$ \\
\hline Citrato de calcio $0.1 \%$ & $\mathrm{~T} 1$ & 3 & $8 \mathrm{~kg}$ & $120 \mathrm{~g}$ & $24 \mathrm{~kg}$ & $300 \mathrm{~g}$ \\
\hline Citrato de calcio $0.2 \%$ & $\mathrm{~T} 2$ & 3 & $8 \mathrm{~kg}$ & $120 \mathrm{~g}$ & $24 \mathrm{~kg}$ & $300 \mathrm{~g}$ \\
\hline Citrato de calcio $0.3 \%$ & $\mathrm{~T} 3$ & 3 & $8 \mathrm{~kg}$ & $120 \mathrm{~g}$ & $24 \mathrm{~kg}$ & $300 \mathrm{~g}$ \\
\hline Total & & & & & $96 \mathrm{~kg}$ & $1200 \mathrm{~g}$ \\
\hline
\end{tabular}

TUE $^{*}$ El tamaño de la unidad experimental.

Para el control de los parámetros bromatológicos se tomaron muestras de $120 \mathrm{~g}$ y fueron enviadas al Laboratorio de Análisis Ambiental e Inspección Lab.CESTTA, mientras que el control microbiológico se realizó en el Laboratorio de Microbiología de los Alimentos y Técnicas Industriales de la Facultad de Ciencias Pecuarias de la ESPOCH. Para los resultados organolépticos, se seleccionó el panel de catadores que calificó las salchichas bajo los siguientes parámetros: apariencia 10 puntos, color 10 puntos, sabor 10 puntos, aroma 10 puntos, textura 10 puntos, sobre un total 50 puntos.

\section{Resultados.}

\section{Calidad nutritiva}

Las medias del contenido de humedad en las salchichas Frankfurt no presentaron diferencias estadísticas ( $\mathrm{P}>0.05)$, por efecto de la utilización de los diferentes niveles de citrato de calcio obtenido de las cáscaras del huevo y empleado como antioxidante, así como por efecto del eritorbato de sodio (tratamiento control), por cuanto el contenido de humedad en las salchichas fluctuó entre 64.05 y $64.23 \%$, en aquellas elaboradas con 0.2 y $0.1 \%$, en su orden, estableciéndose una media general de $64.14+2.02 \%$, lo que permite deducir que al adicionar un antioxidante natural, el contenido de humedad del embutido no se altera, por 
cuanto los antioxidantes según Bueno, M. (2009), son sustancias que se adicionan a los alimentos para evitar el enranciamiento, por cuanto la oxidación no solo produce pérdidas nutritivas, sino que además suele generar un sabor desagradable que hace rechazar el alimento.

Tomando como referencia la elaboración de salchichas vienesa, se puede indicar que los resultados obtenidos en el presente trabajo, guardan relación con los estudios de Moreno, G. (2001) y Martínez, N. (2004), quienes registraron contenidos entre 64 y $66 \%$, cuando utilizaron almidones en reemplazo de la carne de res, a pesar de que los productos utilizados por estos investigadores tienen la capacidad de absorber y mantener el agua, pero que en todos los casos los valores señalados se encuentran dentro de los requisitos exigidos por el INEN (1996), en la Norma NTE INEN 1 338:96, donde se indica que la salchicha escaldada o cocida debe contener hasta un $65 \%$ de humedad (pérdida por calentamiento).

Las medias del contenido de materia seca por ser inversamente proporcional al contenido de humedad, no presentaron diferencias estadísticas entre sí ( $\mathrm{P}>0.05)$, por cuanto los valores encontrados variaron entre 35.77 y $35.95 \%$ en las salchichas elaboradas con el empleo de 0.1 y $0.2 \%$ de citrato de calcio obtenido de las cáscaras del huevo y empleado como antioxidante, respectivamente, estableciéndose un contenido de materia seca promedio en la salchicha Frankfurt de 35.86+2.02 \%, valores reportados en el cuadro 3, que guardan relación con los estudios de Moreno, G. (2001), Martínez, N. (2004). Quienes reportaron contenidos de materia seca de 34 y $36 \%$, en su orden, ratificándose que los aditivos antioxidantes no alteran el contenido de materia seca, sino que previenen el deterioro de las grasas y los pigmentos de la carne

Cuadro 3 Composición nutritiva y fisicoquímica de las salchichas Frankfurt elaboradas con diferentes niveles de citrato de calcio obtenido de cáscaras de huevo y utilizado como antioxidante natural.

\begin{tabular}{|c|c|c|c|c|c|c|c|c|c|c|c|c|}
\hline \multirow[b]{2}{*}{ Parámetro } & \multicolumn{8}{|c|}{ Niveles de Citrato de calcio, $\%$} & \multirow{2}{*}{$\begin{array}{l}\text { Media } \\
\text { general }\end{array}$} & \multirow{2}{*}{$\begin{array}{l}\text { Desviación } \\
\text { estándar }\end{array}$} & \multirow[b]{2}{*}{ Prob. } & \multirow[b]{2}{*}{$b^{*}$} \\
\hline & 0 & & 0,1 & & 0,2 & & 0,3 & & & & & \\
\hline $\begin{array}{l}\text { Características } \\
\text { nutritivas }\end{array}$ & & & & & & & & & & & & \\
\hline Humedad, \% & 64,15 & a & 64,23 & $\mathrm{a}$ & 64,05 & a & 64,18 & $\mathrm{a}$ & 64,14 & 2,017 & 0,999 & \\
\hline Materia seca, $\%$ & 35,89 & a & 35,77 & a & 35,95 & a & 35,82 & $\mathrm{a}$ & 35,86 & 2,017 & 0,999 & \\
\hline Proteína, \% & 14,85 & $\mathrm{a}$ & 14,74 & a & 14,80 & a & 15,04 & $\mathrm{a}$ & 14,86 & 0,534 & 0,815 & \\
\hline Grasa, \% & 17,16 & $\mathrm{a}$ & 17,31 & $\mathrm{a}$ & 17,22 & $\mathrm{a}$ & 17,10 & $\mathrm{a}$ & 17,20 & 2,679 & 0,999 & \\
\hline Cenizas, $\%$ & 3,27 & a & 3,33 & a & 3,47 & $\mathrm{a}$ & 3,42 & $\mathrm{a}$ & 3,37 & 0,397 & 0,851 & \\
\hline Físico-químicas & & & & & & & & & & & & \\
\hline $\begin{array}{l}\mathrm{pH} \text { inicial } \\
\text { Acidez inicial, }\end{array}$ & 6,05 & $\mathrm{~b}$ & 6,05 & $\mathrm{~b}$ & 6,17 & $\mathrm{a}$ & 6,18 & $\mathrm{a}$ & 6,11 & 0,080 & 0,000 & 0,52 \\
\hline${ }^{\circ} \mathrm{D}$ & 9,63 & $\mathrm{~b}$ & 9,67 & $\mathrm{~b}$ & 9,85 & $\mathrm{a}$ & 9,85 & $\mathrm{a}$ & 9,75 & 0,177 & 0,038 & 0,83 \\
\hline
\end{tabular}


Vida de anaquel:

pH a los 15 días $\quad 6,10$ b $\quad 6,12$ b 6,22 a $\quad 6,25$ a $6,17 \quad 0,081 \quad 0,000 \quad 0,55$

Acidez a los 15

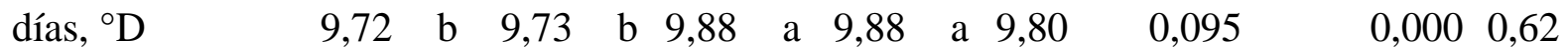

Referencia del Laboratorio Lab.CESTTA y Laboratorio de Microbiología de los Alimentos y Técnicas Industriales de la Facultad de Ciencias Pecuarias de la ESPOCH

Prob. > 0,05: No existen diferencias estadísticas.

Prob.<0,05: Existen diferencias significativas

Prob.< 0,01: Existen diferencias altamente significativas

b*: Coeficiente de regresión

Promedios con letras distintas en una misma fila difieren estadísticamente de acuerdo a la prueba de Waller-Duncan.

Los contenidos de proteína de las salchichas Frankfurt elaboradas con diferentes niveles de citrato de calcio obtenido de las cáscaras del huevo y empleado como antioxidante, así como por efecto del eritorbato de sodio (tratamiento control), no registran diferencias estadísticas ( $\mathrm{P}>0.05$ ), por cuanto los valores encontrados fueron de 14.74 a $15.04 \%$ cuando se utilizaron 0.1 y $0.3 \%$ de citrato de calcio, respectivamente, estableciéndose una media general de $14.86+0.53 \%$, por lo que puede indicarse que el citrato de calcio o eritorbato de sodio utilizados como antioxidantes no afectan el contenido proteico de la salchicha, por cuanto son productos antioxidantes, Colores orgánicos, embutidos que ofrecen un auténtico placer, ingredientes aromáticos y más, afirma industriaalimenticia.(2016), el beneficio más obvio del antioxidante natural en comparación con el artificial, es que el natural proporciona una declaración de ingrediente en la etiqueta más favorable, dejando de ser una verdadera preocupación para los servicios de comidas, ya que en los momentos actuales los consumidores prefieren alimentos elaborados con productos considerados como naturales antes que con productos químicos.

Los valores determinados son superiores a las exigencias requeridas por el INEN (1996), en su Norma NTE INEN 1 338:96, donde se indica que la salchicha escaldada debe contener como mínimo un $12 \%$, por cuanto en el presente trabajo se supera el $14 \%$ de proteína, al igual que en reporte de Moreno, G. (2001) y Martínez, N. (2004), Donde se señala que la salchicha Frankfurt presenta un aporte proteico del 13.1 \%; en cambio guardan relación con los estudios de Moreno, G. (2001) y Martínez, N. (2004), que reportan contenidos de proteína de 15.43 y $14.06 \%$ en las salchichas elaboradas a base de carne de bovino y cerdo (salchicha vienesa), por lo tanto la salchicha Frankfurt presenta un aporte proteico similar que las salchichas de los estudios citados.

El contenido de grasa de las salchichas Frankfurt no presentaron diferencias estadísticas ( $\mathrm{P}>0.05)$, por efecto de la utilización de los diferentes niveles de citrato de calcio obtenido de las cáscaras del huevo y empleado como antioxidante, así como por efecto del eritorbato 
de sodio (tratamiento control), encontrándose contenidos entre $17.10 \%$ en la salchicha elaborada con $0.3 \%$ y $17.31 \%$ en las elaboradas con $0.1 \%$ citrato de calcio, por lo que se establece un promedio de $17.20+2.68 \%$, valores que son óptimos para la alimentación, lo que se contrapone con lo señalado en Salchichas cocidas tipo Frankfurt Diferentes en calidad cárnica, grasa y calorías en revista.consumer.(2014), donde se reporta que las salchichas Frankfurt son alimentos grasos y de un considerable contenido de sodio, por lo que su consumo debe restringirse en caso de sobrepeso u obesidad; diferencia que puede deberse posiblemente a las formulaciones empleadas, por cuanto en estudios realizados a nivel nacional en salchichas escaldadas, entre los que se mencionan a Moreno, G. (2001) y Martínez, N. (2004), determinaron contenidos de grasa entre 16.1 y $18.8 \%$; además, los resultados obtenidos demuestran que se encuentran por debajo del límite máximo permitido por el INEN (1996), en la Norma NTE INEN 1 338:96, donde se indica que el contenido máximo de grasa total para productos escaldados debe ser de $25 \%$. Notándose adicionalmente que el efecto antioxidante del citrato de calcio y del eritorbato de sodio, previnieron la oxidación de las grasas, ya que no se cambiaron las características del alimento en cuanto a su sabor, color, olor, debiéndose tener en cuenta en cambio que es muy importante la estabilidad según el pH del alimento al que se adicionan, para que no sufran reacciones químicas que eviten su función (Ospina, J. 2001).

Al igual que los otros nutrientes evaluados, el contenido de cenizas de la salchicha Frankfurt no presentaron diferencias estadísticas $(\mathrm{P}>0.05)$ entre las medias registradas de los diferentes tratamientos, registrándose una media general de $3.37+0.40 \%$, por cuanto con los valores determinados variaron entre 3.27 y $3.42 \%$, que corresponden a las salchichas elaboradas con eritorbato de sodio y con el $0.2 \%$ de citrato de calcio, respectivamente, notándose numéricamente que con el empleo del citrato de calcio se elevó ligeramente el contenido de cenizas que con eritorbato, lo que puede deberse a que el citrato de calcio obtenido con el jugo de limón, este contiene casi 30 componentes antioxidantes, además de su riqueza en potasio y calcio Propiedades medicinales de Citrus limón afirma botanical-online.(2016). Si se toma en cuenta el contenido de cenizas encontradas en las salchichas evaluadas y considerando el reporte del INEN (1996), los valores encontrados se encuentran dentro de los requisitos establecidos, por cuanto en esta norma se señala que en los productos embutidos escaldados el contenido de cenizas, no debe sobrepasar del $5 \%$.

\section{Valoración Físico Química}

\section{pH}

Los valores medios del $\mathrm{pH}$ inicial de las salchichas Frankfurt procesadas con eritorbato de sodio y $0.1 \%$ de citrato de calcio (6.05 en ambos casos), presentaron diferencias altamente significativas $(\mathrm{P}<0.01)$, respecto a las medias encontradas en las salchichas que se elaboraron con 0.2 y 0.3 de citrato de calcio ya que presentaron valores de $\mathrm{pH}$ de 6.17 y 6.18 , por lo que mediante el análisis de la regresión se estableció una tendencia lineal altamente significativa 
que determina que por cada unidad adicional del citrato de calcio hasta el $0.3 \%$, el pH de salchicha se incrementa en 0.52 unidades. Indicándose que los valores de $\mathrm{pH}$ determinados se encuentran dentro de los requisitos exigidos por el INEN (1996), por cuanto en esta norma se señala que el pH máximo permitido para productos embutidos escaldados es de 6.2.

\section{Acidez}

Las salchichas Frankfurt obtenidas al emplearse el eritorbato de sodio y $0.1 \%$ de citrato de calcio presentaron valores de acidez de 9.63 y $9.67{ }^{\circ} \mathrm{D}$, respectivamente, valores que presentan diferencias significativas $(\mathrm{P}<0.05)$, con los determinados en las salchichas elaboradas con 0.2 y $0.3 \%$ de citrato de calcio $\left(9.85^{\circ} \mathrm{D}\right.$, en ambos casos), por lo que el análisis de la regresión estableció una tendencia lineal altamente significativa, que determina que por cada unidad adicional del citrato de calcio hasta $0.3 \%$, la acidez de la salchicha se incrementa en 0.83 unidades, Pero en todo caso, el empleo de 0.2 y $0.3 \%$ favorecen la acidez normal de las salchichas Frankfurt para impedir el desarrollo microbiano.

\section{Valoración Microbiológica.}

De los resultados reportados por el Laboratorio de Microbiología de los Alimentos y Técnicas Industriales de la Facultad de Ciencias Pecuarias, con respecto a los análisis microbiológicos realizados en las salchichas Frankfurt elaboradas con diferentes niveles de citrato de calcio obtenido de las cáscaras del huevo y empleado como antioxidante, así como por efecto del eritorbato de sodio (tratamiento control), se puede indicar que las salchichas no presentaron microorganismos del tipo aerobios mesófilos así como de coliformes, por cuanto en todas las muestras analizadas se reportan valores negativos (ausencia), para estas bacterias

\section{Valoración Organoléptica}

Las respuestas obtenidas de la valoración organoléptica de la salchicha Frankfurt, no fueron diferentes estadísticamente entre las medias de los diferentes parámetros evaluados lo que indica que la utilización del citrato de calcio obtenido de las cáscaras del huevo y empleado como antioxidante, así como por efecto del eritorbato de sodio (tratamiento control) no afectaron las propiedades evaluadas.

Las medias de la valoración de la apariencia del producto, no fueron estadísticamente diferentes $(\mathrm{F} \&<\mathrm{F}$ tab) por efecto de la utilización del eritorbato de sodio (tratamiento control) como de los diferentes niveles del citrato de calcio obtenido de las cáscaras del huevo y empleado, por cuanto las calificaciones alcanzadas variaron entre 8.62 y 8.98 puntos sobre 10 de referencia, y que corresponden a las salchichas elaboradas con $0.3 \%$ de citrato de calcio y con el eritorbato de sodio, respectivamente, debido posiblemente a que en todos los casos se pudo apreciar una pasta compacta y uniforme, de color rosado, es decir, respecto a lo que señala Picallo, A. (2002) que generalmente la apariencia se detecta a través de la vista 
que comprende el color, el brillo, la forma y puede dar una idea de textura; sumándose a esto el efecto favorable que obtiene mediante el proceso de escaldado.

El color de las salchichas Frankfurt estadísticamente fueron similares ( $F \&<F$ tab) por efecto de la utilización del eritorbato de sodio (tratamiento control) como de los diferentes niveles del citrato de calcio, por cuanto las valoraciones asignadas fueron entre 8.67 y 8.87 puntos sobre 10 de referencia, debido a que en todos los casos se observaron una coloración rojiza característica, debido al proceso de escaldado, añadiendo a esto el efecto de los nitritos que producen una mejor pigmentación de la carne curada (Lawrie, H. 2000).

Las medias de la valoración del sabor que presentaron las salchichas Frankfurt, no fueron diferentes estadísticamente ( $\mathrm{F} \&<\mathrm{F}$ tab), pues alcanzaron puntuaciones de 8.90 a 9.07 puntos sobre 10 de referencia, sin establecerse algún efecto beneficioso del citrato de calcio sobre el eritorbato de sodio, utilizados como antioxidantes, por cuanto Wirth, F. (2001), indica que los sabores agradables de los productos cárnicos, se derivan de la grasa, la misma que no se alteró por efecto de los antioxidantes evaluados,

Partiendo de lo que señala Lima, J. (2017), donde se indica que el aroma es la propiedad organoléptica que presentan algunas sustancias que pueden ser percibidas por inhalación en la cavidad buco nasal. Hay sustancias que pueden ser aromáticas para unas personas y no para otras, ya que la percepción depende de la intensidad, tipo y variedad; que normalmente no pueden ser valoradas de forma cuantitativa, ni tampoco hay una clasificación de olores básicos, por lo que las características del aroma que desprendían las salchichas Frankfurt, al ser valoradas en base a la preferencia del panel de cata, las respuestas asignadas no presentaron diferencias estadísticas $(\mathrm{F} \&<\mathrm{F}$ tab), por efecto de los niveles de citrato de calcio y del eritorbato de sodio empleados, por cuanto las calificaciones asignadas fueron entre 8.60 y 9.05 puntos sobre 10 de referencia, puntuaciones que pueden deberse a lo que menciona Forrest, J. (1989), en que la carne es muy susceptible a la absorción de materias volátiles, es mediante el escaldado que se contribuye a mejorar el aroma de la salchicha pues al evitar que hidrocarburos y formaldehído se desprenden del alimento se mejora notablemente dicha característica. (Lima, J. 2017).

La textura de las salchichas Frankfurt, no se vio influenciada estadísticamente (F\& $<\mathrm{F}$ tab), por efecto los niveles de citrato de calcio y del eritorbato de sodio empleados, pues recibieron calificaciones entre 7.52 y 8.80 puntos sobre 10 puntos de referencia, que corresponden a las elaboradas con $0.3 \%$ de citrato de calcio y la otra con eritorbato de sodio, respectivamente; aunque numéricamente se aprecia una ligera preferencia por la última, lo que puede deberse a lo que señala Lawrie, H. (2000), quien al hablar sobre la textura de la carne indica que el consumidor considera que la textura y la dureza son las propiedades más importantes de la calidad organoléptica, anteponiéndolas incluso al sabor y al color, a pesar de lo difícil que resulta definir cada término. La sensación de dureza se debe en primer lugar a la facilidad con que los dientes penetran en la carne, en segundo lugar a la facilidad con que la carne se 
divide fragmentos y en tercer lugar a la cantidad de residuo que queda después de la masticación, siento esta sensación procedente de la grasa, la misma que se evitó su deterioro con la utilización de los antioxidantes evaluados.

\section{Valoración Total}

La valoración total de las características organolépticas no registraron diferencias estadísticas en las puntuaciones totales alcanzadas, ya que los valores asignados fluctuaron entre 42.85 y 44.18 puntos sobre 50 puntos de referencia, que corresponden a las salchichas Frankfurt elaboradas con $0.3 \%$ de citrato de calcio y con eritorbato de sodio, respectivamente, por lo que de acuerdo a la escala de valoración de los alimentos de Witting, E. (1981), les corresponden calificaciones de Muy Buenas a todos los grupos, lo que denota que la utilización del citrato de calcio en reemplazo del eritorbato de sodio como antioxidante natural, en la elaboración de salchicha Frankfurt no afecta las características organolépticas, siendo un producto altamente aceptado por parte de los consumidores y que fue representado a través de los catadores que evaluaron este producto.

\section{Vida de Anaquel}

\section{pH a los 15 días}

Los valores del $\mathrm{pH}$ a los 15 días de almacenamiento de las salchichas Frankfurt elaboradas con eritorbato y $0.1 \%$ de citrato de calcio (6.10 y 6.12, en su orden), presentaron diferencias altamente significativas $(\mathrm{P}<0.01)$, con las medias registradas en las salchichas que se elaboraron con 0.2 y 0.3 de citrato de calcio por cuanto presentaron valores de $\mathrm{pH}$ de 6.22 y 6.25, respectivamente, por lo que a través del análisis de la regresión se estableció una tendencia lineal altamente significativa, que determina que por cada unidad adicional del citrato de calcio hasta el $0.3 \%$, el pH de salchicha se incrementa en 0.55 unidades; lo que puede deberse a que el citrato de calcio tienen propiedades antioxidantes transferidas por el limón, por lo que se pone de manifiesto lo señalado por Lima, J. (2009), quien indica que los valores bajos de pH pueden ayudar en la conservación de los alimentos de dos maneras: directamente, inhibiendo el crecimiento microbiano, e indirectamente, a base de disminuir la resistencia al calor de los microorganismos, en los alimentos que vayan a ser tratados térmicamente. Por otra parte, los valores determinados del $\mathrm{pH}$ se encuentran entre los márgenes adecuados exigidos por el INEN (1996), por cuanto en esta norma se señala que el pH máximo permitido para productos embutidos escaldados es de 6.2.

\section{Acidez a los 15 días}

La acidez a los 15 días de almacenamiento de las salchichas Frankfurt obtenidas al emplearse el eritorbato de sodio y $0.1 \%$ de citrato de calcio fue de 9.72 y $9.73{ }^{\circ} \mathrm{D}$, respectivamente, valores que estadísticamente presentan diferencias altamente significativas $(\mathrm{P}<0.01)$, con las medias determinadas en las salchichas elaboradas con 0.2 y $0.3 \%$ de citrato de calcio que 
fueron de $9.88^{\circ} \mathrm{D}$, en ambos casos, por lo que el análisis de la regresión estableció una tendencia lineal altamente significativa, que determina que por cada unidad adicional del citrato de calcio hasta $0.3 \%$, la acidez de la salchicha se incrementa en 0.62 unidades; lo que puede deberse a que para obtener el citrato de calcio se utiliza jugo de limón, que es muy rico en vitamina $\mathrm{C}$, además contiene numerosos flavonoides que tienen propiedades antioxidantes, capaces de prevenir la actividad negativa de los radicales libres (Moreno, G. 2001)

\section{Evaluación Económica}

De acuerdo a los resultados reportados, se determina que cuando se emplea el eritorbato de sodio, el costo de producción por kg de salchicha Frankfurt es de 3.72 dólares, en cambio que con el citrato de calcio independientemente de la cantidad (hasta el $0.3 \%$ ), estos costos fueron de 3.68 dólares, estableciéndose un ahorro de 4 centavos de dólar por kg producido, con relación al análisis del beneficio/costo se determinó que al emplear el eritorbato de sodio, se obtiene un beneficio/costo de 1.29, que representa que por cada dólar invertido se obtiene una rentabilidad de 29 centavos de dólar (29 \%) por cada parada producida, en cambio con la utilización del citrato de calcio entre 0.1 y $0.3 \%$ su rentabilidad se incrementa al $31 \%$, o lo que es lo mismo un Beneficio/costo de 1.31, que representa que por cada dólar invertido se obtiene una rentabilidad de 31 centavos de dólar (31\%) por cada parada producida, cantidad hasta el $0.3 \%$ de citrato de calcio.

\section{Conclusiones.}

- El empleo de los diferentes niveles de citrato de calcio obtenido de las cáscaras de huevo como antioxidante en la elaboración de salchicha Frankfurt, no presentó diferencias estadísticas en la composición nutritiva, presentando valores son superiores a los requisitos exigidos por el INEN (1996), en su Norma NTE INEN 1 338:96.

- La salchicha Frankfurt presenta contenidos de 64.14+0.02\% de humedad, $14.86+0.53 \%$ de proteína, $17.20+2.68 \%$ de grasa y $3.37+0.40 \%$ de cenizas.

- Las propiedades físicas de la salchicha como son el pH y la acidez determinaron tendencias lineales tanto al inicio como en la evaluación a los 15 días de almacenamiento, que determina que a medida que se incrementa los niveles del citrato de calcio hasta el $0.3 \%$, estos tienden a incrementarse, pero sin sobrepasar los requisitos exigidos por el INEN (1996), ya que los valores promedios iníciales de $\mathrm{pH}$ y acidez fueron de $6.11+0.8$ y $9.75+0.177{ }^{\circ} \mathrm{D}$ en su orden; y a los 15 días de $6.17+0.08$ y $9.80+0.10{ }^{\circ} \mathrm{D}$.

- Los antioxidantes evaluados no alteraron las características organolépticas como la apariencia del producto, color, sabor, aroma y textura, recibiendo todos las salchichas de los tratamientos evaluados una valoración total de Muy Buenas de acuerdo a la escala de Witting, E. (1981). 
- Con la utilización del citrato de calcio se establece un ahorro de 4 centavos de dólar por $\mathrm{kg}$ producido con respecto al eritorbato de sodio (3.68 frente a 3.72 dólares $/ \mathrm{kg}$ ), por lo que se alcanzó una rentabilidad del $31 \%$ por lote producido (cada 15 días).

Por lo que se recomienda:

- Tomando en consideración el análisis económico se recomienda elaborar salchicha Frankfurt con la utilización del citrato de calcio obtenido de las cáscaras de huevo como antioxidante, por cuanto se reducen los costos de producción, se eleva la rentabilidad, sin que se altere la calidad nutritiva, microbiológica y organoléptica.

- Replicar el presente estudio pero incrementando los niveles de citrato de calcio obtenido de las cáscaras de huevo como antioxidante, por cuanto además del efecto antioxidante se estaría añadiendo otras características como fuente de vitaminas $\mathrm{C}$ y minerales como el calcio, los mismos que las personas los consumen como suplementos alimenticios para la prevención de determinadas enfermedades.

- Evaluar la utilización del citrato de calcio obtenido de las cáscaras de huevo como antioxidante, en otros productos cárnicos en los que se emplean cantidades considerables de grasa animal, como la mortadela, queso de chancho, salchichones, entre otros.

\section{Referencias bibliográficas.}

BUENO, M (2018) Aditivos antioxidantes. Biosalud-Instituto de Medicina Biológica y Antienvejecimiento Recuperado el 20 Marzo de 2019 http://www.biosalud.org. 2018 . . Aditivos antioxidantes. Biosalud-Instituto de Medicina Biológica y Antienvejecimiento

Colores orgánicos, embutidos que ofrecen un auténtico placer, ingredientes aromáticos y más (2016) Recuperado el 15 de mayo de 2019 https://www.industriaalimenticia.com/ articles/89873-colores-org\%C3\%A1nicos-embutidos-que-ofrecen-un-aut\%C3\%A9nticoplacer-ingredientes-arom\%C3\%A1ticos-y-m\%C3\%A1s

ECUADOR, INSTITUTO NACIONAL ECUATORIANO DE NORMALIZACIÓN (INEN). 1996. Norma NTE INEN1 344:96. Quito, Ecuador.

FORREST, J. 1989. Fundamentos de la ciencia de la carne. sn. Zaragoza, España.Edit. ACRIBIA. pp 41-43.

Información nutricional: Salchicha tipo Frankfurt una nueva alternativa de consumo (2012). Recuperado el 20 de mayo 2019 https://lareiras.gal/es/?s=salchicha+frankfut 
La proteína láctea caseína como antioxidante se utiliza en los embutidos (2014) Recuperado 11 Abril de 2019. http://www.infocarne.com /noticias/2019/7/7176_ caseína_ se_convierte_embutidos_primera_empresa_española_au.asp

LAWRIE, H. 2002. Ciencia de la carne. 2a ed. Zaragoza, España. Edit ACRIBIA. pp 1025.

LIMA, J. (2017) pH y acidez. Recuperado el 20 de Marzo http://www.elergonomista.com. 2017. Alimentación. Estudio de los alimentos, Aroma.

MARTÍNEZ, N. 2004. Evaluación de cuatro niveles $(1.25,2.5,3.75$ y 5.0\%) de fécula de maíz en la elaboración de salchicha vienesa. Tesis de Grado. Facultad de Ciencias Pecuarias, ESPOCH. Riobamba, Ecuador. pp 35-57.

MIRA, J. 1998. Compendio de Ciencia y Tecnología de la carne. $1^{\text {a }}$ ed. Ed. AASI. Riobamba, Ecuador. pp. 10, 32

MORENO, G. 2001. Utilización de fécula de papa en la elaboración de salchicha vienesa. Tesis de Grado. Facultad de Ciencias Pecuarias, ESPOCH. Riobamba, Ecuador. pp $38-57$.

OSPINA, J. 2001. Enciclopedia Agropecuaria Terranova. 2a ed Tomo 5: Ingeniería y agroindustria. Bogota, Colombia. Edit. Terranova. pp 320-326.

Propiedades medicinales de Citrus limón (2016) Recuperado el 19 de Marzo 2019 https://www.botanical-online.com/alimentos/limon-propiedades-medicinales

PICALLO, A. 2002. El análisis sensorial como herramienta de calidad de carne y productos cárnicos de cerdo. Buenos Aires, Argentina. Edit. INTA. Página de Internet .pdf.

Salchichas cocidas tipo Frankfurt Diferentes en calidad cárnica, grasa y calorías (2014) Recuperado el 18 de marzo 2019 http://revista.consumer.es/web/es /20041101/actualidad /analisis 1/69295.php

WIRTH, F. 2001. Valores normativos de la tecnología de la carne. sn. Zaragoza, España. Edit. Acribia. pp 38-40.

WITTING, E. 1981. Evaluación sensorial. Una metodología actual para tecnología de alimentos. sn. Santiago, Chile. Edit. Talleres gráficos USACH. pp 4-10.

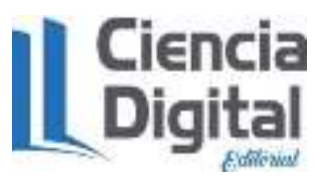




\section{PARA CITAR EL ARTÍCULO INDEXADO.}

Guzmán Acán, F., Bonifaz Ramos, V. de los Ángeles, \& Esparza Bonilla, C. (2019). Caracterización bromatología, microbiológica y sensorial de la salchicha Frankfurt elaborada con Citrato de Calcio como antioxidante natural. Ciencia Digital, 3(3), 473-487. https://doi.org/10.33262/cienciadigital.v3i3.663

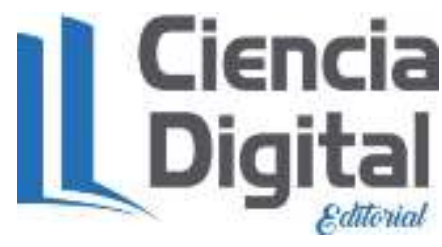

El artículo que se publica es de exclusiva responsabilidad de los autores y no necesariamente reflejan el pensamiento de la Revista Ciencia Digital.

El artículo queda en propiedad de la revista y, por tanto, su publicación parcial y/o total en otro medio tiene que ser autorizado por el director de la Revista Ciencia Digital.
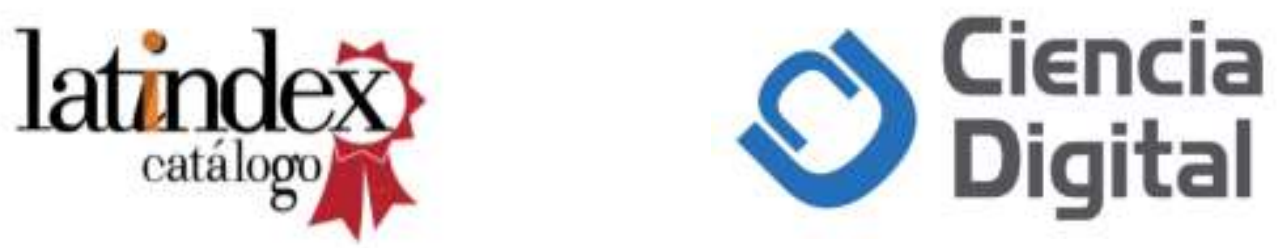